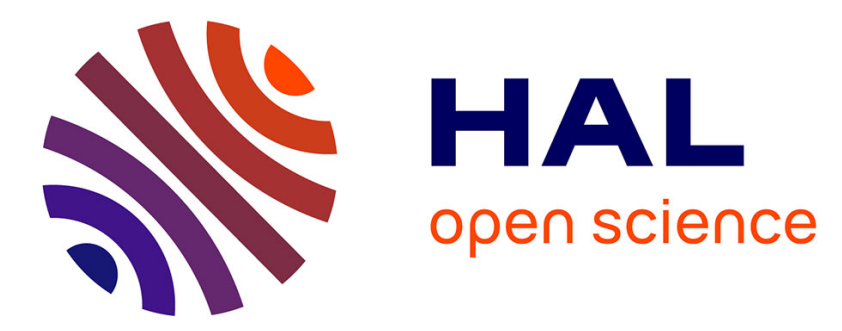

\title{
Le pont de Barbegal au vallon des Arcs à Fontvieille (Bouches-du-Rhône) : étude archéologique de la dérivation de l'aqueduc d'Arles
}

Philippe Leveau, Robert Thernot

\section{- To cite this version:}

Philippe Leveau, Robert Thernot. Le pont de Barbegal au vallon des Arcs à Fontvieille (Bouches-duRhône) : étude archéologique de la dérivation de l'aqueduc d'Arles. Gallia - Archéologie de la France antique, 2005, Aqueducs de la Gaule méditerranéenne, 62, pp.97-105. 10.3406/galia.2005.3223 . hal01912625

\section{HAL Id: hal-01912625 \\ https://hal.science/hal-01912625}

Submitted on 14 Jan 2020

HAL is a multi-disciplinary open access archive for the deposit and dissemination of scientific research documents, whether they are published or not. The documents may come from teaching and research institutions in France or abroad, or from public or private research centers.
L'archive ouverte pluridisciplinaire HAL, est destinée au dépôt et à la diffusion de documents scientifiques de niveau recherche, publiés ou non, émanant des établissements d'enseignement et de recherche français ou étrangers, des laboratoires publics ou privés.

\section{(ㅇ)(1) $\$$}

Distributed under a Creative Commons Attribution - NonCommercial - NoDerivatives| 4.0 


\title{
LE PONT DE BARBEGAL AU VALLON DES ARCS À FONTVIEILLE (BOUCHES-DU-RHÔNE)
}

\author{
Étude archéologique de la dérivation de l'aqueduc d'Arles
}

\author{
Philippe LEVEAU et Robert THERnOT
}

\begin{abstract}
Mots-clés. Période romaine, Arles, aqueiucs, architecture, hydraulique, techniques de construrtion.
Résumé. En relation uvec la construction des moulins de Barbegal, un pont-aqueduc fut consiruit pour assurer l'alimentation hydraulique de l'usine par la branche méridionale de l'aqueduc d'Arles. L'ouvrage comporte deux parties : une section sur mur plein en opus caementicium à un parement en opus vittatum, une section de 28 arches construiles dans la contimuité du mur plein suivant les mêmes techniques. Les arches reposent sur des piliers dont limposte et la base sont constituées par des blocs de molasse de grande laille. L'une des arches conservée présente la trace d'une reconstruction, probablement à la suite d'une destruction volontaire. Une évaluation du débit de l'outrage est proposée.
\end{abstract}

Key-words. Roman period, Arelate, aqueduct, architecture, hydraulics, construction techniques.

Abstract. In connection with the construction of the Barbegal mills, a suspended aqueduct was built for the water-supply of the factory by the means of the southern branch of the aqueduct of Arles. It is composed of two parts: one with solid masonry walls in opus caementicium with an opus vittatum facing, the other with 28 arches built with the same techniques in the continuity of the masonry wall. The impost and the basis of the piers which support the arches are made of large mollasse blocks. On one of the arches being preserved, a mark from a rebuilding presumably following a voluntary destruction. An estimation of the flow is given here.

Translation : Isabelle FACI)LT:T

Schlagwörter. Römische Epoche, Arles, rïmische Wasserleitungen, Architektur; Wasserbau, Bautechniken.

Zusammenfassung. Im Anschluß an den Bau der Mühlen von Barbegal wurde ein Aquädukt errichtet, um die Wasserversorgung des Betriebes über den südlichen Strang der Wasserleitung von Arles zu sichern. Das Bauwerk besteht aus zwei Teilen, dem in opus vittatum verblendeten Mauerwerkskörper aus opus caementitium, sowie 28 Bögen, die in Verlängerung des Mauerwerks in derselben Technik errichtet sind. Die Bögen ruhen auf Pfeilern, deren Kämpfer und Sockel aus großformatigen. Molassequadern bestehen. Einer der erhaltenen Bögen zweist Spuren einer Reparatur auf, die möglicherweise nach einer gezielten Zerstörung nötig wurde. In dem Beitrag wird pine Schätzung der Leistung der Wasserleitung zur Diskussion gestellt.

Überselzung : Stefan WIRTH

L.c bassin amont des ponts du vallon des Arcs, dont J.-L. Guendon présente dans ce dossier le fonctionnement à partir des dépôts carbonatés (voir supra, p. 87-96), a vu sa fonction changer avec la construction du pont-aqueduc étudié ici. La découverte d'une dérivation à l'amont de ce bassin a permis d'expliquer la relation existant entre les branches nord ct sud de l'aqueduc des Alpilles. Elle a montré qu'une partic des eaux, jusqu'alors affectées à l'alimentation de la ville d'Arles, avait changé de destination et d'usage à la suite de la construction d'un nouvel ouvrage de franchissement élevé à l'est du précédent pour alimenter l'usine de Barbegal en énergie hydraulique (fig. 86).
En 1989, au début des fouilles, le pont-aqueduc de Barbegal n'était observable qu'en un petit nombre de points, essentiellement à ses deux extrémités aval et amont. Au nord de la route qui traverse d'est en ouest la double ligne des aqueducs, entre celle-ci et le canal des Alpilles, il émergeait des broussailles sur près de $80 \mathrm{~m}$. Complètement invisible au sud sous des accumulations de cailloux, il réapparaissait sous la forme d'un mur plein d'une cinquantaine de mètres de long à l'amont de la Pierre Trouée, passage creusé dans le chaînon de la Pène pour lui permettre d'atteindre les moulins. On comprend les hésitations des archéologues face à des vestiges indistincts dont la chronologie ne leur apparaissait pas. L..-A. Constans avait 


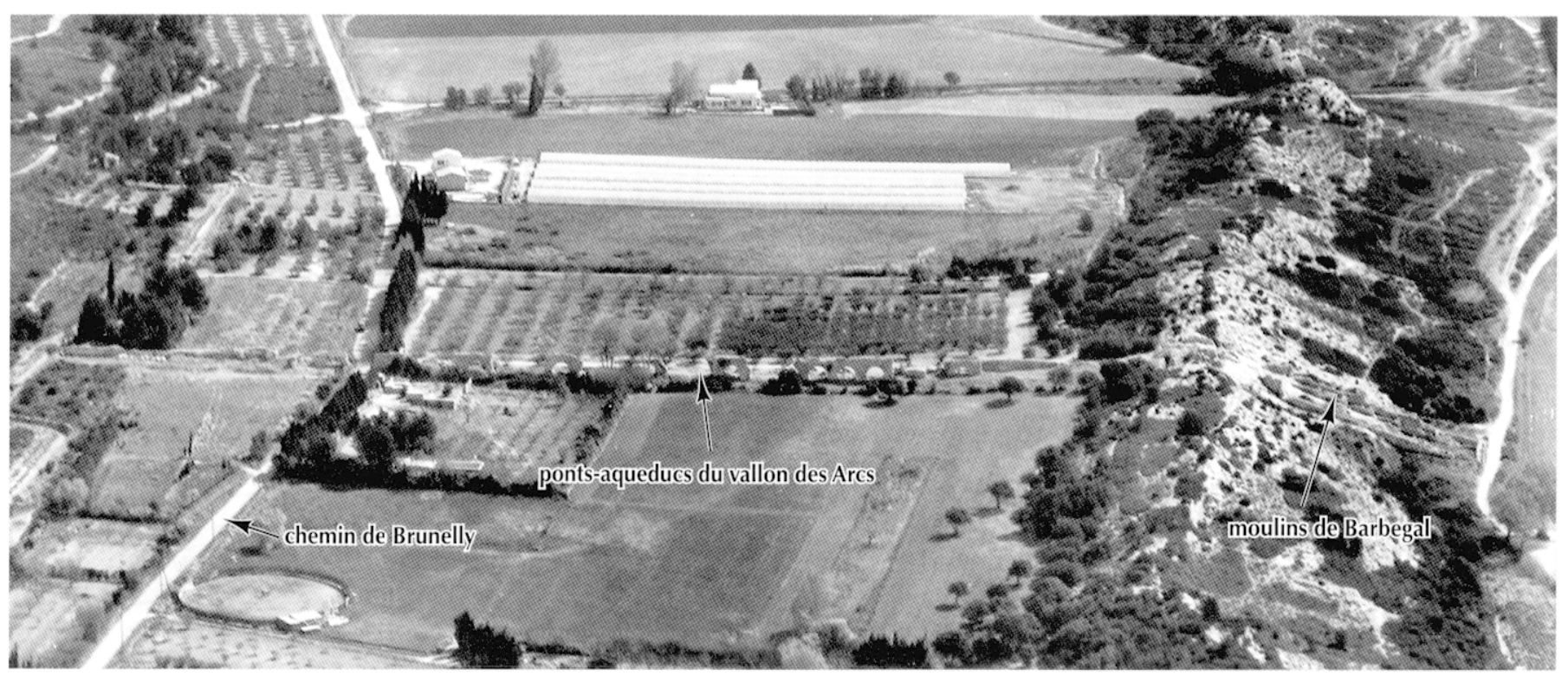

Fig. 86 - Vue aérienne du pont-aqueduc et des moulins de Barbegal à Fontvieille, Bouches-du-Rhône (cliché A. Chenet, CNRS).

bien vu que les deux ouvrages n'avaient pas la même pente, mais il interprétait les vestiges des moulins comme ceux d'un siphon. Postérieurement aux fouilles de F. Benoit qui en avait établi la fonction véritable, $\mathrm{A}$. Grenier se demandait s'il ne fallait pas rechercher " la trace d'unc ancienne réunion des deux aqueducs " juste à l'amont du " coude brusque qu'après les arcades jumelées des deux aqueducs, la conduite d'Eygalières prononce vers l'ouest tandis que celle de Caparon continue vers le réservoir de la meunerie "(Grenier, 1960, p. 81). Une telle proposition pouvait s'appuyer sur le cas du pont de Jouy sur l'aqueduc de Metz qui porte, lui aussi, une double canalisation débouchant sur un bassin (id., ibid., p. 205). Dans ces conditions, seule une fouille pouvait permettre de trancher entre plusieurs hypothèses. C'est pour cette raison que nous avons débuté la fouille de ce côté avant de rechercher un bassin à l'amont.

Les dégagements qui ont permis de dresser un plan des ouvrages n'ont pas été complets. Dans la section amont, ils n'ont porté que sur la face ouest du monument car il n'a pas été possible d'intervenir dans la propriété qui longe l'ouvrage à l'est. À l'aval, au sud de la route, toute la partie longée par le canal d'irrigation n'a pas été explorée. La fouille a débuté par un sondage entrepris à l'amont du mur qui portait le canal, au niveau de la pile qui a reçu le $n^{\circ} 26$. Les dégagements se sont étendus sur une cinquantaine de mètres entre la culée aval et le point où le canal d'irrigation traverse les vestiges. Ils ont mis au jour le haut de cinq piles et les restes de la dernière arche (effondrée).

\section{L'ARCHITECTURE DU PONT-AQUEDUC}

Le nouveau pont (conventionnellement appelé ici pont B) se calquait sur un premier ouvrage, le pont de l'aqueduc d'Arles (pont A), de sorte que l'on est tenté de parler à leur

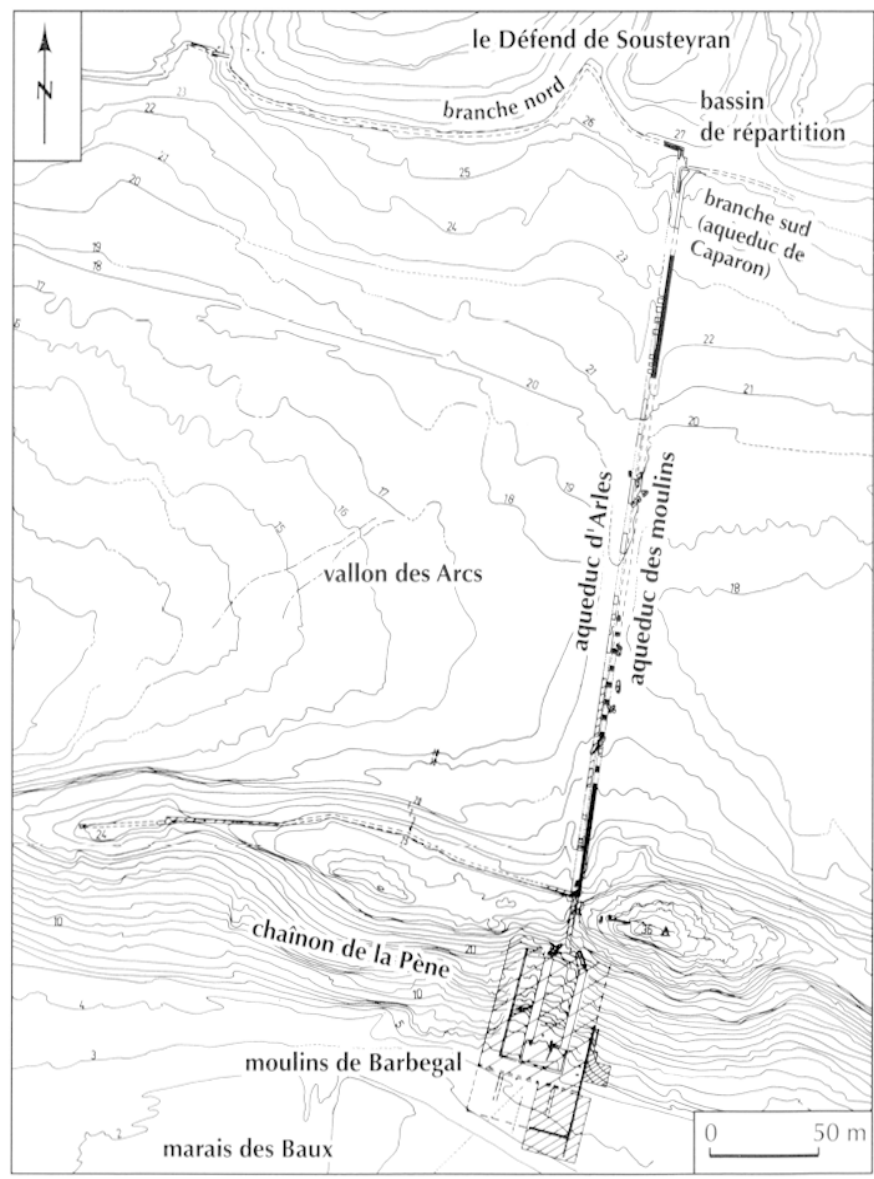

Fig. 87 - Plan des aqueducs au vallon des Ars (dessin M. Borel); CNRS, d'après une photogrammétrie de A. Carrié, CNRS).

propos de "ponts jumeaux" (fig. 87). En réalité, si les piliers des deux ouvrages sont alignés, ils diffèrent par la proportion 
de plein et de vide dans la maçonnerie. Les sections sur mur plein du pont $\mathrm{B}$ sont plus longues que celles du pont $\mathrm{A}$. En effet, alors que la première arche du pont $A$ se trouve à $52 \mathrm{~m}$ du bassin et la dernière à $14 \mathrm{~m}$ du chaînon de la Pène, soit une section pleine de $64,20 \mathrm{~m}$ représentant $20 \%$ de sa longueur totale, la première et la dernière arche du pont $\mathrm{B}$ sont respectivement à $69 \mathrm{~m}$ du bassin et à $52,50 \mathrm{~m}$ du chaînon, ce qui correspond à une longueur cumulée de $121,50 \mathrm{~m}$, soit près de $40 \%$ de la longueur totale $(317,20 \mathrm{~m})$ du franchissement. Cette différence d'appareillage - grand appareil pour le pont A et petit appareil pour le pont B - traduit leur position chronologique dans l'histoire de la construction romaine.

\section{LA SECTION EN MUR PLEIN}

Dans les sections aval et amont de l'ouvrage, le conduit est porté par un mur plein (fig. 88). En général, on considère qu'à partir de $2,50 \mathrm{~m}$ à $3 \mathrm{~m}$, les architectes romains préféraient passer à des arcatures - c'est ce qui a été pratiqué à l'amont (fig. 89). À l'aval, un sondage implanté contre le parement est, à $15 \mathrm{~m}$ de la culée, a montré que ce mur d'une hauteur de
3,60 m au-dessus du talon de fondation avait été élevé sur une fondation de blocage en tranchée débordant de $12 \mathrm{~cm}$ et profonde de $1 \mathrm{~m}$. Conservé en élévation, ce mur présente un parement en opus vittatum, d'aspect homogène, attestant unc source d'approvisionnement unique. La carrière exploitée a livré un calcaire tendre de teinte claire, sensible à l'altération météorique, comme l'indiquent les angles émoussés des moellons. D'une hauteur de 10 à $12 \mathrm{~cm}$, les moellons de 10 à $25 \mathrm{~cm}$ de long et de $25 \mathrm{~cm}$ de profondeur sont taillés systématiquement en dépouille afin d'assurer une bonne liaison avec le blocage. Le mortier garnissant les joints a pratiquement partout disparu, sauf dans la partie amont, à la base du parement occidental du mur, où les joints encore en place sont retracés à la pointe.

Le blocage est formé d'une alternance de lits de moellons bruts et de mortier. Les interstices entre les éléments ne sont pas systématiquement comblés par le liant, de sorte que l'opus caementicium ainsi réalisé présente un aspect vacuolaire. Deux alignements superposés de trous de boulins traversants, visibles en partie sud, ont été ménagés. Le premier est situé à une quarantaine de centimètres du sol actuel, l'autre à $1,20 \mathrm{~m}$ de la
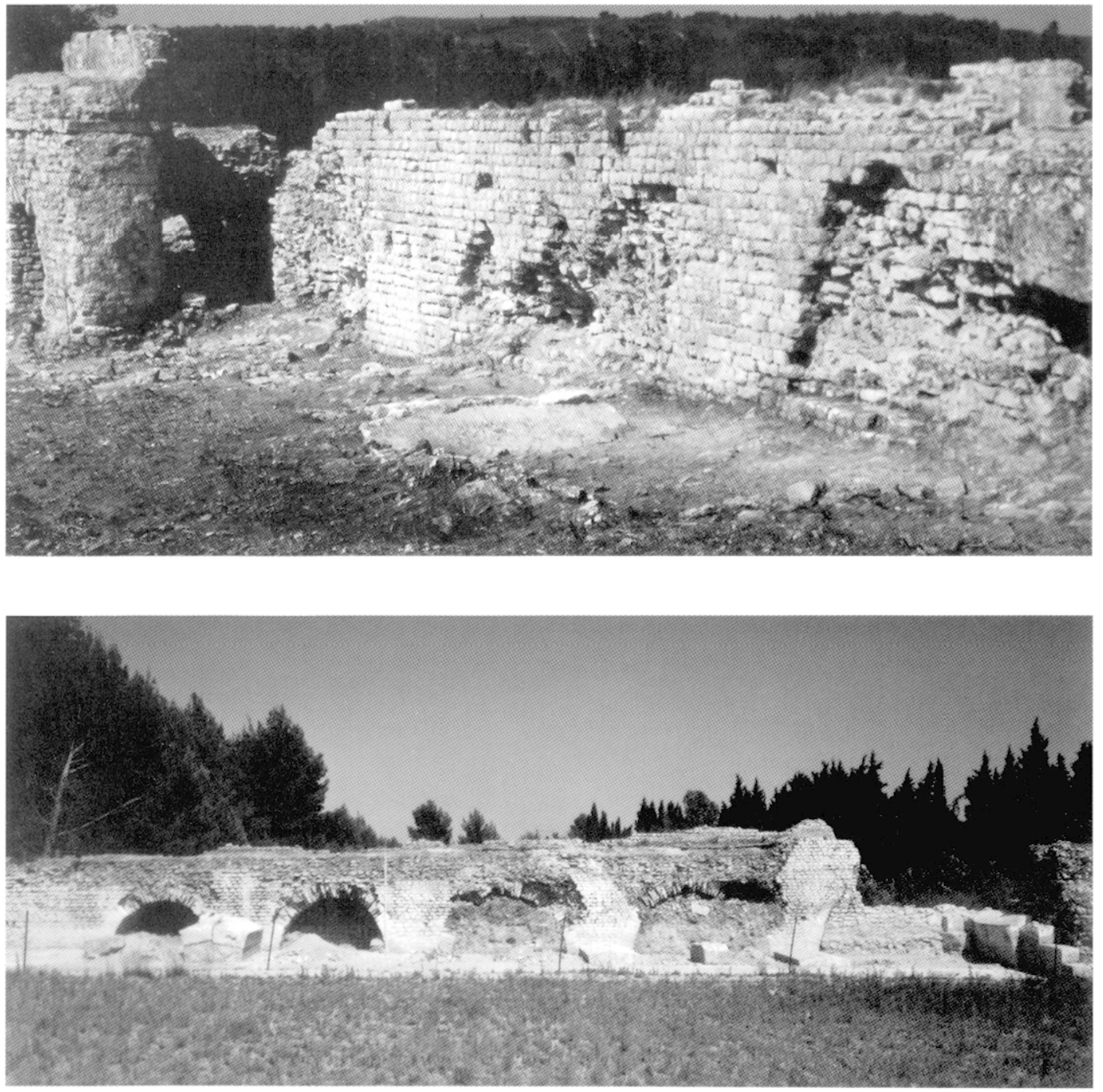

Fig. 88 - Culée aval du parement ouest du pontaqueduc de Barbegal à Fontvieille (cliché P. Leveau, CNRS).
Fig. 89 - Les arches $1-5$ de la section amont du pontaqueduc de Barbegal à Fontrieille (cliché P. Foliot, (.NRS). 
première ligne. Couvert chacun par un moellon de l'assise supérieure disposé en linteau, les orifices occupent la hauteur d'une assise et ne sont pas régulièrement espacés.

Le parement de ce mur a souffert du côté exposé aux vents d'ouest. En témoigne l'état de la façade de ce côté. Au sud, en particulier, les parties où il est bien conservé dessinent le négatif des piliers disparus de l'aqueduc d'Arles.

\section{LA SECTION GENTRALE SUR ARCHES}

La section sur arches compte 27 piles numérotées de Bl à B27, du nord au sud, soit 28 arches.

Seules subsistent en élévation les quatre premières arches amont (fig. 89). Dans la partie centrale, l'espacement entre les piles est de 5,21 m en moyenne. L'écartement entre les piles varie en fonction du niveau du terrain naturel. Dans la partie médiane, les variations de portée d'une arche à l'autre sont compensées par des écarts inverses dans les arches voisines. On relève les valeurs suivantes dans le tableau V. Relativement dégradées, les arches conservées au nord laissent apparaître leur structure interne. Elles sont construites dans la continuité du mur plein qui les précède en amont suivant les mêmes techniques. Les arcs de front sont formés de claveaux soigneusement taillés dans un calcaire gréseux beige clair, formant un rouleau extradossé de $0,45 \mathrm{~m}$ d'épaisseur. L'intrados bénéficie d'un soin comparable dans l'agencement des claveaux (fig. 90).

Les piles de l'aqueduc des moulins sont très dérasées. Leur emprise au sol, proche du carré, varie de $1,90 \mathrm{~m}$ à $2,12 \mathrm{~m}$ en largeur.

Le sondage exploratoire ouvert au début de la campagne de fouilles a permis d'observer le mode de construction de la pile B26 (fig. 92). Trois blocs de molasse de $64 \mathrm{~cm}$ d'épaisseur et débordant de $24 \mathrm{~cm}$, grossièrement équarris, ont été posés directement sur le substrat rocheux, un faciès gréseux du Rognacien (fig. 91, n7). À cet endroit, le rocher très altéré en surface a été régularisé de façon à offrir au bloc de fondation

Tabl. V - Pont-aqueduc de Barbegal: dimension des arches.

\begin{tabular}{|c|c|c|}
\hline Arche & $\begin{array}{c}\text { Portée entre piles } \\
(\mathbf{e n ~} \mathbf{~})\end{array}$ & $\begin{array}{c}\text { Diamètre arc } \\
\text { (en } \mathbf{~})\end{array}$ \\
\hline$B 0-B 1$ & 2,40 & 3,00 \\
\hline$B 1-B 2$ & 3,00 & 3,40 \\
\hline$B 2-B 3$ & 3,70 & 3,70 \\
\hline$B 3-B 4$ & 3,90 & 4,20 \\
\hline$B 19-B 20$ & 5,40 & - \\
\hline$B 20-B 21$ & 5,30 & - \\
\hline$B 21-B 22$ & 5,30 & - \\
\hline$B 22-B 23$ & 5,20 & - \\
\hline$B 23-B 24$ & 5,60 & - \\
\hline$B 24-B 25$ & 4,80 & - \\
\hline$B 25-B 26$ & 5,30 & - \\
\hline$B 26-B 27$ & 4,40 & - \\
\hline$B 27-B 28$ & 5,60 & - \\
\hline
\end{tabular}

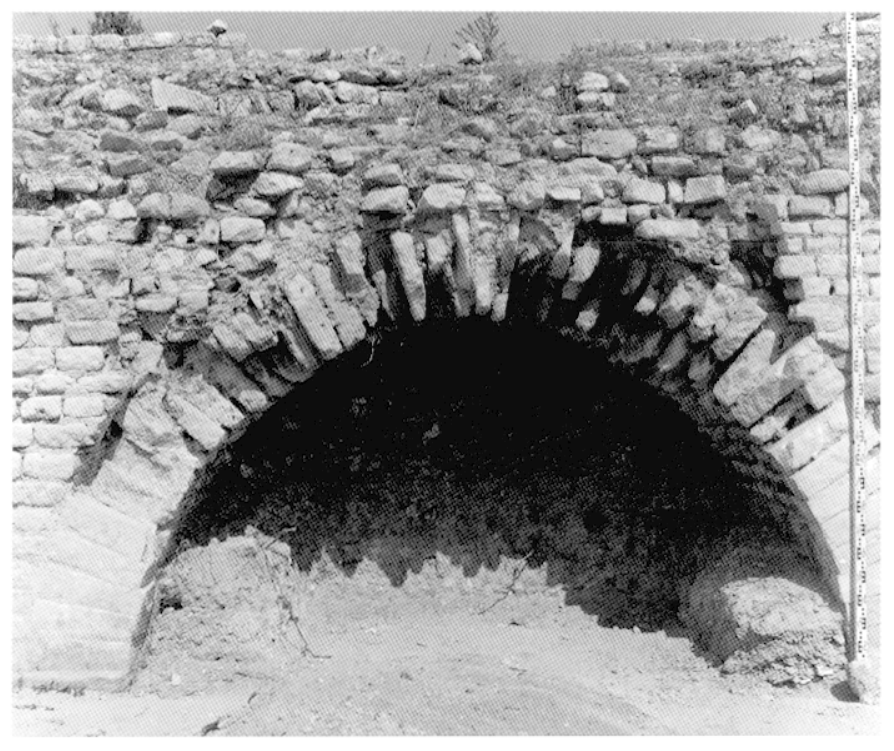

Fig. 90 - Pont-aqueduc de Barbegal à Fontvieille: détail de l'arche B1-B2 (cliché P. Leveau, CNRS).

un lit d'attente horizontal. Le niveau d'installation de cette fondation est situé une cinquantaine de centimètres plus bas que celui de la pile voisine de l'aqueduc d'Arles. Le parement de la pile est formé de neuf assises de moellons. Ces derniers ont été majoritairement tirés d'une roche jaune et poreuse qui a fortement subi les attaques de l'humidité capillaire et atmosphérique. Les moellons, de $12 \mathrm{~cm}$ de haut et de 12 à $20 \mathrm{~cm}$ de long, ont une face de parement sommairement dressée, et l'épaisseur des joints, lissés au fer, varie de 2 à $4 \mathrm{~cm}$. On remarque toutefois que les angles de la pile B25 bénéficient d'un traitement particulier, avec des moellons de meilleure qualité, mieux taillés. Le mortier utilisé ne comporte pas de tuileau. Un autre sondage près de la culée nord, au pied de la pile $\mathrm{Bl}$, montre un hérisson de cailloux sur lequel reposent quatre blocs de fondation débordants, recevant ici directement l'arc.

Le lit d'attente de l'assise d'imposte de la pile B26 est situé $0,70 \mathrm{~m}$ plus bas que celui de l'aqueduc d'Arles (fig. 91 et 92). D'une hauteur de $0,45 \mathrm{~m}$, l'imposte est composée de trois blocs de molasse juxtaposés, à joints vifs, de $0,84 \mathrm{~m}$ de large et de longueur égale ou légèrement supérieure à $2,05 \mathrm{~m}$, soit la largeur de la pile. Les blocs sont disposés perpendiculairement à l'axe du pont. Les chants très érodés n'ont pas gardé de traces d'un traitement formel. Des logements d'agrafes métalliques en forme de double $\mathrm{T}$ sont taillés sur les lits d'attente des blocs de l'imposte. Réunissant deux éléments, les agrafes aujourd'hui disparues, devaient avoir des têtes de $3,20 \mathrm{~cm}$ d'épaisseur, $11 \mathrm{~cm}$ de longueur et $4 \mathrm{~cm}$ de largeur. La barre joignant les têtes avait une largeur de $4 \mathrm{~cm}$. Des traces d'outil autour des logements témoignent du surcreusement que les récupérateurs ont dû pratiquer pour extraire les agrafes. Les encastrements mesurent $0,65 \mathrm{~m}$ de longueur et 15 à $20 \mathrm{~cm}$ de largeur. Un trou de préhension de $11 \mathrm{~cm}$ sur $4 \mathrm{~cm}$ est visible dans la partic médiane des deux blocs orientaux. 


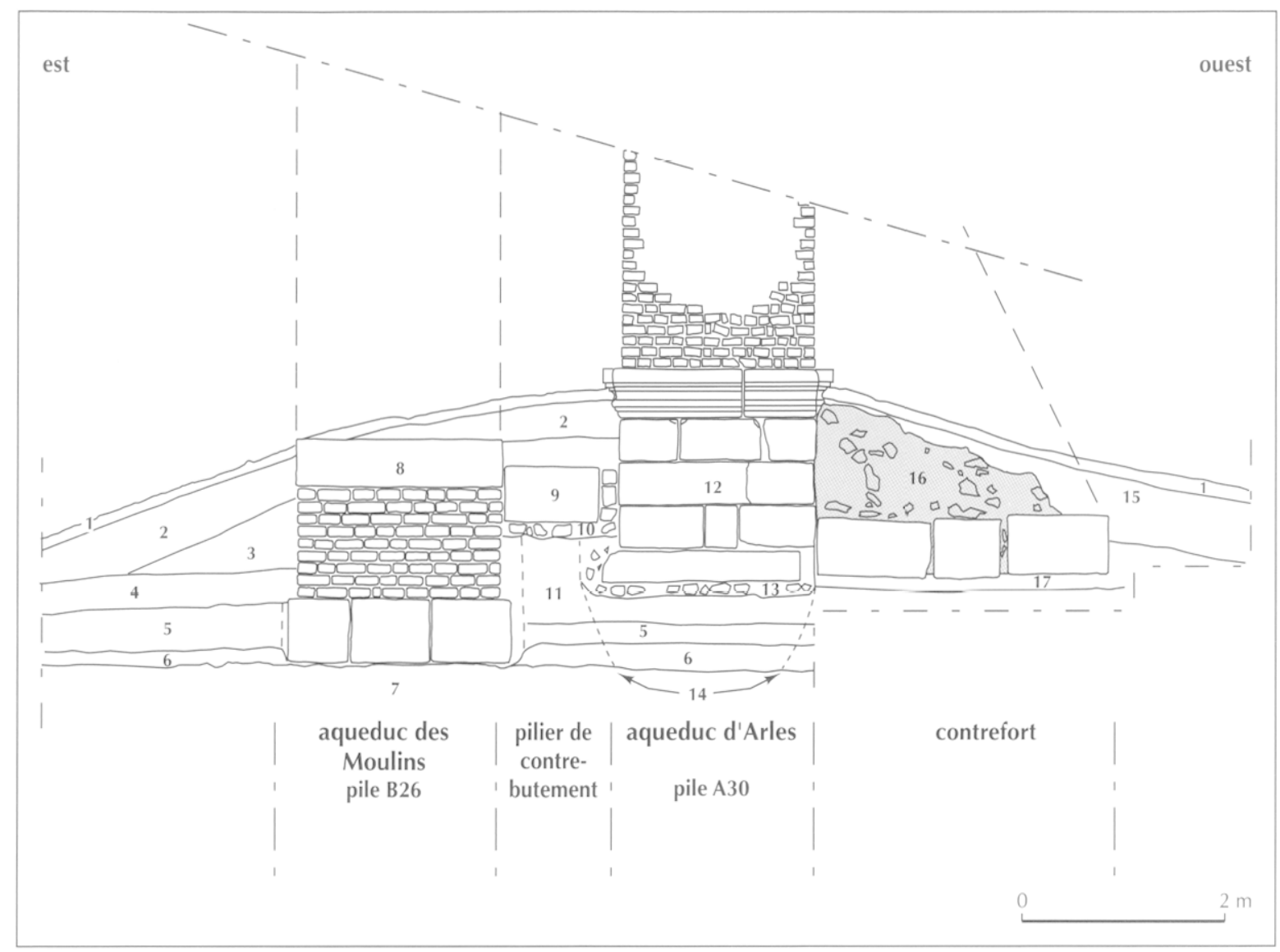

1. terre végétale et débris de maçonnerie

2. couche sableuse avec éléments de démolition et épierrement

3. couche de destruction

4. limon brun

5. colluvions limono-sableuses et cailloutis brun-rouge

6. marne jaune ocre et gravillon (altération du substrat)

7. substrat gréseux (Rognacien)

3. pile de l'aqueduc des moulins

9. pilier de contrebutement

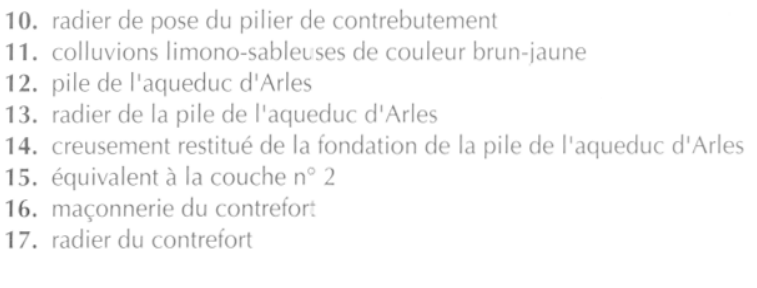

Fig. 91 - Coupe est-ouest sur les deux ponts (DAO R. Thernot, INRAP).

\section{LA CHRONOLOGIE DES OUVRAGES ET LA STRATIGRAPHIE DE LA PILE B26}

L'ouvrage de franchissement du vallon des Arcs a été placé sur le point topographique actuellement le plus haut de l'ensellement qui sépare le chaînon de la Pène des collines du Défend de Sousteyran. Les ponts jumeaux ont été exploités comme carrière. Mais l'écroulement des maçonneries, le rejet sur le site des pierres sorties des champs par les agriculteurs et l'accumulation des sédiments par un effet de barrage par rapport au vent dominant ont sans doute accentué cette position naturelle. C'est ce qu'a montré la coupe ouverte au niveau de la pile B26 en faisant apparaître le substrat rocheux sur lequel se trouve l'ourrage (fig. 91).

Lne coupe est-ouest, perpendiculaire à l'axe des deux aqueducs et prenant en compte les maçonneries, permet d'observer les unités stratigraphiques suivantes:

1. Couche contenant des débris de maçonnerie de blocage mêlés à une terre végétale humifère de quelques centimètres d'épaisseur recouvre l'ensemble. Cette couche, en pente vers l'est, arrive à la hauteur de l'imposte de la pile de l'aquedur d'Arles (A 30).

2. Couche épaisse contenant des débris de maçonncric emballés dans un sédiment gris contenant du sable provenant de la désagrégation des mortiers. Cette couche recouvre totalement le bloc d'imposte de B26. C'est une couche correspondant à une accumulation de matériaux provenant du pont de l'aqueduc d'Arles et de pierres rejetées depuis le champ à l'occasion de sa mise en culture et à leur érolution sur place.

3. La couche 3 correspond au corps de la pile entre les blocs d'imposte et les blocs de fondation. Inclinée vers l'est comme les précédentes, cette couche se développe en biseau sur unc quarantaine de centimètres de hauteur et sur $1,10 \mathrm{~m}$ à l'est. Elle contient des blocs de maçonnerie plus ou moins désagrégés et des cailloutis anguleux qui proviennent de déchets de taille. 
C'est une couche de destruction contenant des fragments d'amphores, en particulier africaines, et quelques fragments de céramiques sigillées (claire africaine A et $\mathrm{D}$, et surtout claire B). Dans la partie du sondage correspondant à l'ouverture des arches, dans l'axe du mur de contrebutement, elle est riche en débris de concrétions (vers le bloc de parement) et en fragments de mortier de tuileau provenant de la destruction d'un des deux canaux.

4. Constituée de limons bruns contenant de la matière organique et de la malacofaune, la couche 4 est en pente vers l'est. Plus épaisse à l'ouest, où elle atteint une quarantaine de centimètres, qu'à l'est (une dizaine de centimètres), elle recouvre les blocs de fondation de la pile. Il s'agit d'une couche qui s'est déposée alors que l'aqueduc était en fonction. Á l'ouest de la pile, il n'en subsiste que des lambeaux conservés sous le hérisson de fondation du massif de maçonnerie reliant les aqueducs.

5. Épaisse d'une cinquantaine de centimètres, la couche 5 est constituée de limons argileux de couleur brun-rouge, d'une matrice sableuse fine et de cailloutis anguleux; elle est fortement décarbonatée. Il s'agit d'une couche naturelle dans laquelle ont été creusées les fondations des deux ponts. À l'ouest, comme la précédente, elle ne subsiste que sous forme de lambeaux entre les deux tranchées de fondation : celle de la pile de l'aqueduc d'Arles et celle du nouvel ouvrage.

6. Épaisse d'une dizaine de centimètres, la couche 6 est constituée de marnes jaune-ocre et de gravillons issus de l'altération des passées gréseuses provenant de l'altération du substrat.

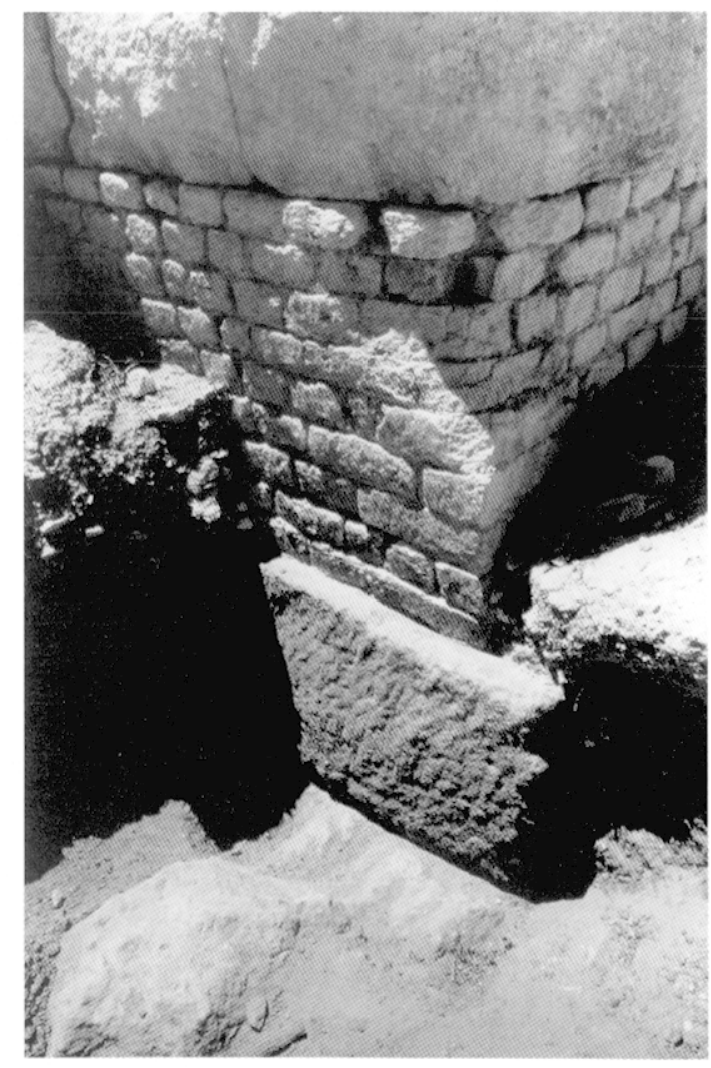

Fig. 92 - Pont-aqueduc de Barbegal à Fontvieille : fouilles du pilier B26 (cliché P. Leveau, CNRS).
7. Tout à fait au fond, à l'est, le sondage atteint le rocher : faciès gréseux du Rognacien.

\section{LA RÉPARATION DE L'ARCHE B4/B5}

Entre le canal des Alpilles et la route, les piles de l'aqueduc d'Arles ont pratiquement disparu, de sorte que l'on peut observer directement la face ouest du pont de Barbegal. Dans ce secteur, entre la culée amont et la pile B4, la conservation du pont est relativement bonne. Sur les arches B2/B3 et B3/B4, les claveaux situés près de la naissance nord des arcs de front sont décalés, en saillie les uns par rapport aux autres, mais le mortier qui les lie ne présente aucune fissure. Cette anomalie semble dès lors résulter d'un glissement des éléments en cours de construction lorsque le mortier n'avait pas encore fait prise.

À partir de la pile B4, ce qui subsiste de l'ouvrage montre que l'arche B4/B5 s'est effondrée dans sa totalité et qu'elle a été entièrement remplacée par un mur plein parementé en moellons taillés, aux assises irrégulières (fig. 93, $\mathrm{n}^{\circ} 1$ ). Ce mur reposant sur une fondation creusée depuis un niveau supérieur de $0,90 \mathrm{~m}$ au niveau de construction initiale, on peut conjecturer qu'il s'agit d'une réfection tardive. Cette maçonnerie forme un redan au contact de la pile voisine (A9) de l'aqueduc d'Arles, destiné à contreforter les ouvrages à l'instar des piliers de contrebutement intercalés entre chaque couple de piles à l'aval. À sa base, on observe la présence d'un bloc courbe qui pourrait provenir d'une tholos de mausolée. C'est dans le même secteur qu'a été trouvé un chapiteau de pilier " corinthisant " (Fournier, 2000, p. 207-208). Nul doute qu'une fouille dans le secteur des piles B6 et B7 apporterait des données importantes sur la chronologie relative des restaurations. Une rupture non provoquée de l'arche pendant la durée d'utilisation de l'ouvrage est surprenante, car le sol dur est à faible profondeur. Celle d'une destruction volontaire est à envisager ; on l'aurait détruite en même temps que celle de l'aqueduc d'Arles pour arrêter l'approvisionnement en eau de la ville. Une telle action n'est envisageable qu'à une époque tardive, par exemple durant les nombreux sièges que la ville a connus pendant le règne de Constantin III (408-411) (Heijmans, 2004, p. 43-78). D’autres hypothèses sont possibles.

L'utilisation de blocs de récupération pour le remplacement de l'arche $\mathrm{B} 5 / \mathrm{B} 6$ est à mettre en parallèle avec les remplois présents dans les contreforts de l'aqueduc d'Arles (fig. 93, $\mathrm{n}^{\circ}$ 2). Il n'est pas exclu que ces aménagements aient été mis en place simultanément lors d'une campagne de restauration du fonctionnement des deux ponts (fig. 93, $n^{\circ} 3$ ).

Au-delà de la route et jusqu'à la culée aval, le pont a disparu en élévation. Dans la majorité des cas, les éléments de maçonneric des arches que l'on peut encore observer gisent à l'est. On ne peut rien conclure de cette disposition qui s'explique par la présence, à l'ouest, de l'aqueduc d'Arles. Cependant, des éléments effondrés de la demière arche et les restes de la pile 1327 semblent indiquer que la rupture de l'arche a été provoquée par des fissures au niveau de la clé de voute. Celle-ci s'est rompuc et la pile B27 a basculé dans son axe (fig. 94). La désagrégation des maçonneries serait donc la cause principale de la disparition de cet ouvage, les carriers n'intervenant que dans un second temps pour récupérer les blocs d'imposte. 

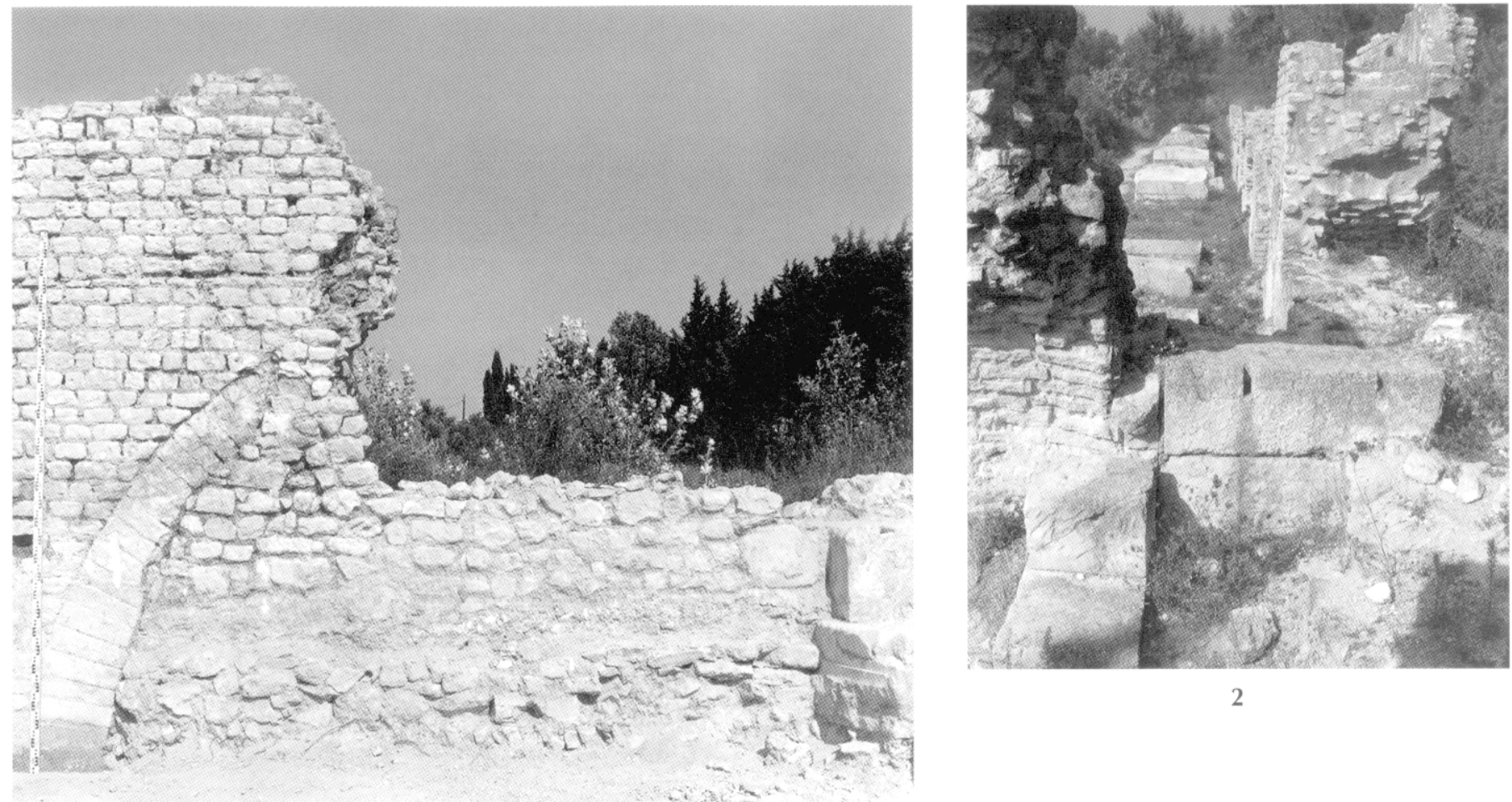

2

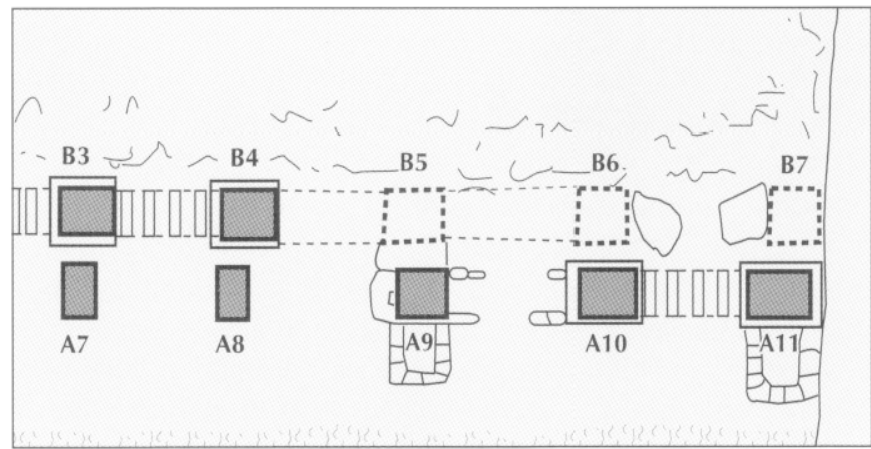

3

Fig. 93 - Réparation de l'arche B4/B5: 1, faşade ouest du pontaqueduc de Barbegal à Fontvieille; 2, photo des deux ouvrages prise du sud : à gauche, l'aqueduc d'Arles et, à droite, l'aqueduc de Barbegal à Fontvieille; 3, plan du secteur: piles A7-A11 et B3-B7 (1, 2, clichés P. Leveau, CNRS ; 3, dessin M. Kleniec et A. Carrié, CNRS).

Fig. 94 - Le specus du pont-aqueduc de Barbegal à Fontvieille : section basculée par effondrement de la dernière arche (B27/culée avial) (cliché P. Foliot, CNRS).

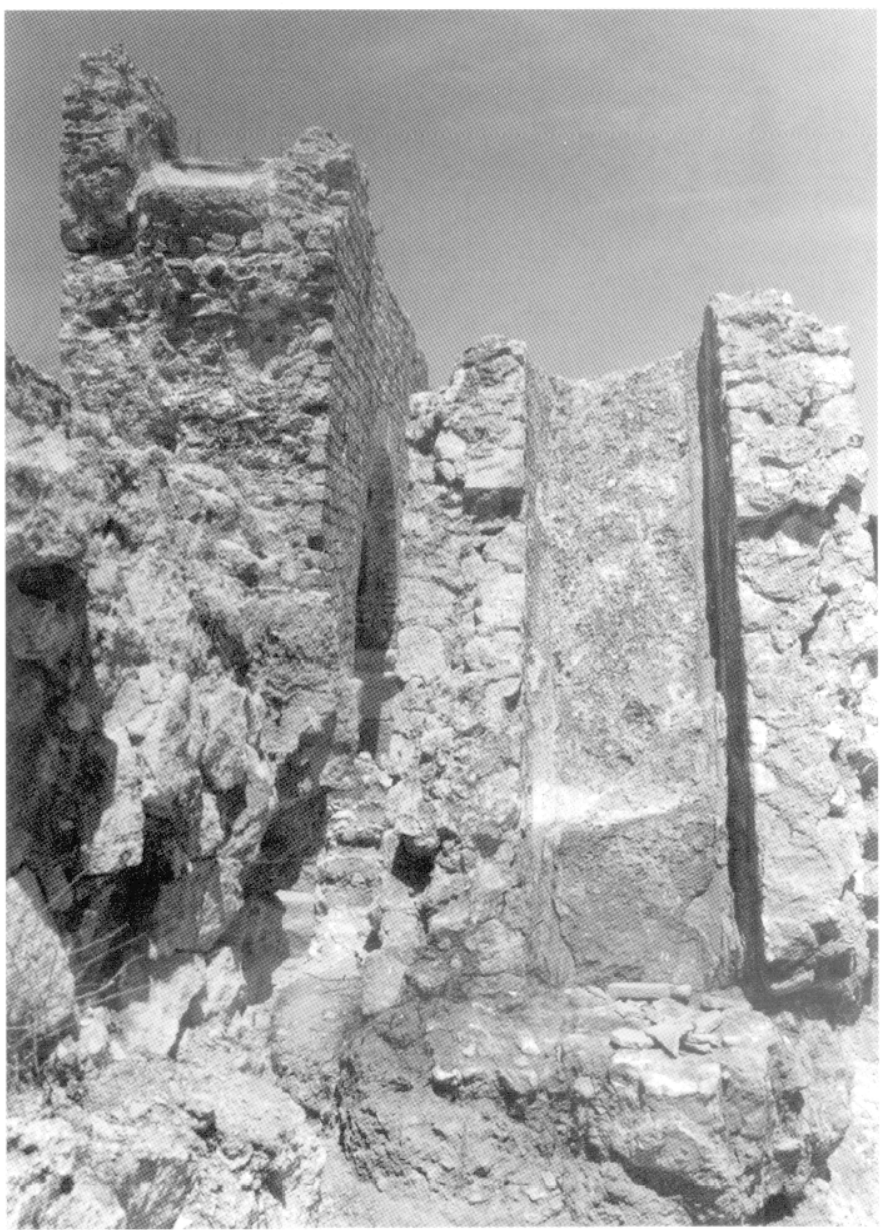




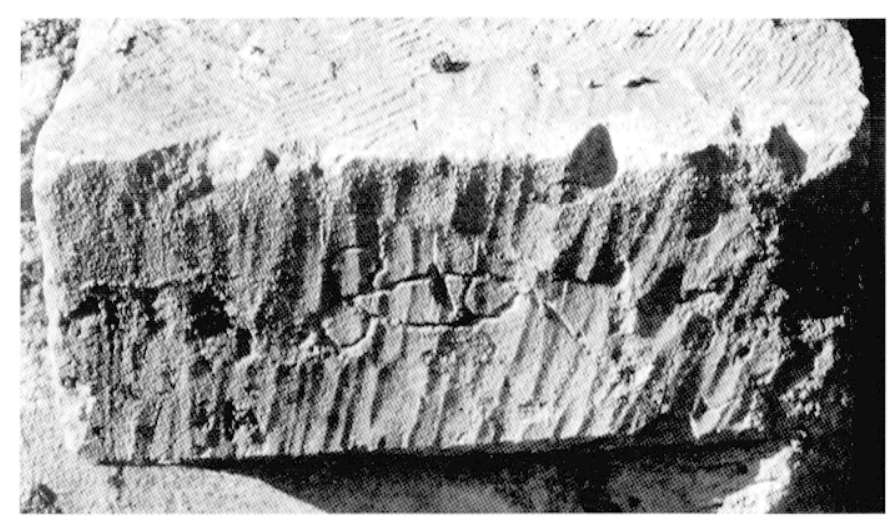

Fig. 95 - Signe phallique (cliché P. Leveau, CNRS).

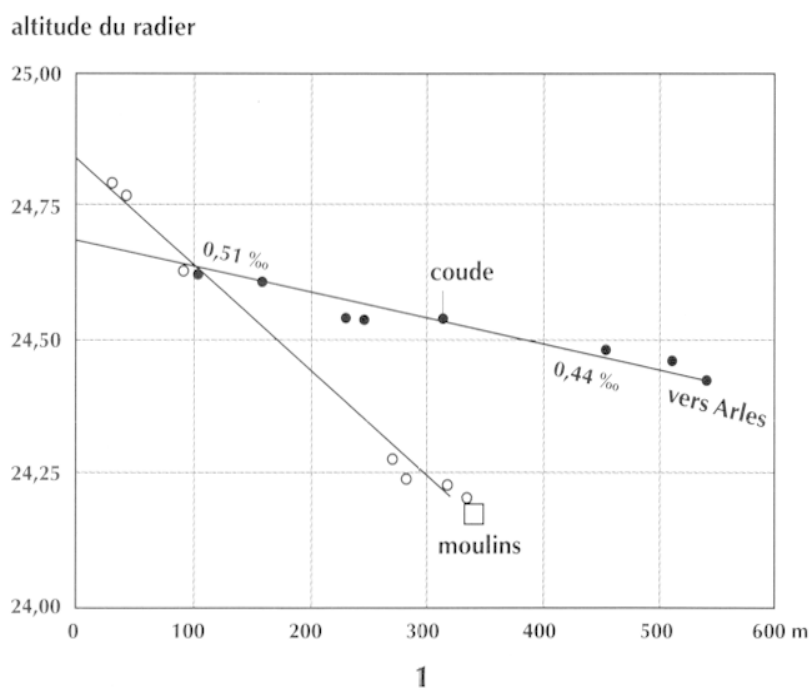

hauteur de remplissage $(\mathrm{m})$

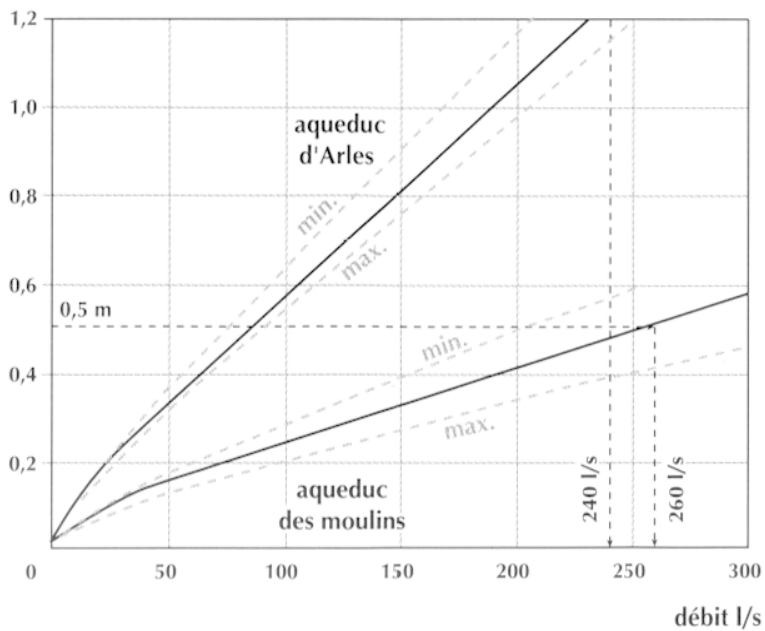

2

Fig. 96 - Diagrammes : a, de la pente des deux ponts; $b$, du débit des aquedurs d'A rles et de Barhegal (IDAO V. Dumas, CNRS d'après Heimann et al., 1993).

\section{LE CONDUIT}

\section{LA PRISE D'EAU}

La fouille de 1989 a montré qu'à $6 \mathrm{~m}$ de son débouché dans le bassin de convergence, le conduit de l'aqueduc de Caparon a été coupé et détourné vers les moulins. Vers l'ouest, du côté du bassin, un mur épais de $0,80 \mathrm{~m}$ et constitué de sept assises de moellons (h. : $0,90 \mathrm{~m}$ ) matérialise la séparation des conduits. Construit sur les concrétions du plancher du premier canal, il est enduit sur sa seule face occidentale (du côté du bassin).

Une brèche encadrée de deux blocs de grande dimension formant piédroits (section à la base : $0,70 \mathrm{~m}$ sur $0,62 \mathrm{~m}$ ) a été ouverte sur le mur gauche (sud) du canal pour assurer la prise d'eau. J.-L. Guendon a observé que la mise en place du bloc de l'angle ouest avait amené à entailler les concrétions de la première génération correspondant à la période de fonctionnement du bassin de convergence (voir J.-I.. Guendon, supra, p. 90-92). Sur la face intérieure du bloc ouest figure un phallus (long. : $20 \mathrm{~cm}$ ), signe dont le caractère apotropaique est bien connu (fig. 95). Pour éviter des turbulences, l'angle du bloc qui lui fait face et fait saillie a été recoupé et enduit de mortier de tuileau. À son départ, le plancher du conduit de la dérivation a été relevé d'une vingtaine de centimètres par rapport à celui du canal sud.

Le nouveau conduit fait deux coudes : un premier, de $55^{\circ}$ vers le sud, puis un second, de $35^{\circ}$, pour prendre un tracé parallèle au conduit de l'aqueduc d'Arles, à $0,70 \mathrm{~m}$ de celui-ci (fig. 96, n' 1). À l'aval, l'écartement est plus important : au niveau de la pile $\mathrm{B} 26$, il s'élève à $1,10 \mathrm{~m}$. Le nouveau canal est encadré par deux murs bajoyers de $0,65 \mathrm{~m}$ construits en opus caementicium. Sa profondeur est de l'ordre de $1 \mathrm{~m}$. Mais la mauvaise conservation du conduit en élévation empêche d'observer d'éventuelles variations de profondeur. Sa largeur interne est d'environ $0,82 \mathrm{~m}$. Elle se situe entre un minimum de $0,79 \mathrm{~m}$ et un maximum de $0,86 \mathrm{~m}$ dans le franchissement de la Pierre Trouée, tranchée de $22 \mathrm{~m}$ de long entaillant le sommet calcaire du chaînon pour atteindre la meunerie hydraulique de Barbegal située sur le versant méridional du relief.

\section{LE MODE DE CONSTRUCTION DU SPECUS}

Le fond du nouveau conduit est constitué d'un radier de béton de tuileau d'une épaisseur de $25 \mathrm{~cm}$ installé sur une couche de mortier de chaux de $3 \mathrm{~cm}$. Un enduit de mortier de tuileau de 3 à $4 \mathrm{~cm}$ d'épaisseur, formé de deux couches, l'une plus grossière, l'autre plus finc, tapisse les parois des piédroits. Dans les angles, des solins d'étanchéité en quart-de-rond complètent le dispositif. Un dosage a montré que le pourcentage de chaux contenu était du même ordre dans le radier du canal que dans celui du bassin de convergence, soit $47 \%$, ce qui confirme la simultanéité des deux opérations de réfection du bassin et de construction de la dérivation. Des concrétions calcaires se sont formées sur le fond et les parois du conduit. D'autres dépôts sont visibles sur les parements des murs pleins amont et aval, révélant des fuites dans le conduit. 
Les caractéristiques de ce conduit le différencient nettement de celui de l'aqueduc des Baux sur lequel il se branche. Ce dernier mesure $1,15 \mathrm{~m}$ de hauteur et $0,82 \mathrm{~m}$ de large. Sa base est constituée d'une structure en L', en béton de chaux coulé, dont les parois latérales sont surmontées de deux murs comptant quatre assises irrégulières. Comme les specus construits selon cette méthode, celui-ci ne comporte ni enduit de tuileau ni solin. Lne voûte de blocage en assure la protection. Ces différences dans la technique de construction des conduits traduisent à la fois l'intervention d'équipes diverses et la chronologie des ouvrages.

\section{LES PENTES ET LE DÉBIT}

En 1992, à la suite de la fouille des ponts et en parallèle avec elle, le débit des canaux a fait l'objet d'une étude spécifique conduite par une équipe d'hydrauliciens allemands du Leichtreiss Institut de Braunschweig (Heimann et al., 1993).

Dans le tableau VI, sont récapitulées les mesures altimétriques qui ont permis l'évaluation du débit proposée. Elles sont, bien entendu, totalement dépendantes de l'état de conservation du pont. Celui-ci n'est satisfaisant que sur les sections amont et aval qui ont été élevées en maçonneries pleines et sur les premières arches du pont où des mesures ont pu être effectuées. Sur le pont lui-même, les dimensions du specus sont extrapolées à partir de mesures faites sur le petit nombre de sections effectivement observées.

Les mesures de pente ont montré qu'immédiatement à l'aval de la dérivation, sur une trentaine de mètres, la pente du nouveau conduit reste faible ou nulle. Au-delà, elle s'accentue pour atteindre $0,85 \mathrm{~m}$ pour $308 \mathrm{~m}$ soit $1,97 \mathrm{~m}$ pour $1 \mathrm{~km}$, ce qui correspond à une valeur beaucoup plus forte que celle du conduit parallèle de l'aqueduc (fig. 96, $\mathrm{n}^{\circ} 2$ ). Cette valeur reste bien inférieure à la pente maximale de 2,11 m que les manuels modernes recommandent pour des cailloux agglomérés. Mais elle est bien supérieure à la pente de $1 \mathrm{~m} / \mathrm{km}$ que l'on observe

Tabl. VI - Pont-aqueduc de Barbegal : altimétrie du radier du specus de l'aqueduc.

\begin{tabular}{|c|c|c|c|}
\hline $\begin{array}{c}\text { Station } \\
\mathrm{n}^{\circ}\end{array}$ & $\begin{array}{l}\text { Distance de } \\
\text { la dérivation } \\
(\mathrm{en} \mathrm{m})\end{array}$ & $\begin{array}{c}\text { Altitude } \\
\text { NGF } \\
\text { (en m) }\end{array}$ & $\begin{array}{l}\text { Caractéristiques du specus } \\
\text { (dimensions en } \mathrm{m} \text { ) }\end{array}$ \\
\hline 1 & 1,90 & 24,71 & long. : 0,$82 ;$ h. : 1,01 \\
\hline 2 & 25,96 & 24,72 & bord droit (est) endommagé; h. conservée : 0,27 \\
\hline 3 & 41,05 & 24,70 & bord droit (est) endommagé; h. conservée : 0,60 \\
\hline 4 & 92,75 & 24,57 & bord droit (est) endommagé ; h. conservée : 0,08 \\
\hline 5 & 267,41 & 24,22 & long. : 0,$83 ;$ h. : $>0,03$ \\
\hline 6 & 282,22 & 24,17 & long. : 0,$79 ;$ h. : $>0,39$ \\
\hline 7 & 317,71 & 24,16 & long. : 0,$81 ;$ h. : $>0,28$ \\
\hline 8 & 330,97 & 24,137 & "Pierre Trouée »; long. : 0,$86 ;$ h. : $>0,14$ \\
\hline
\end{tabular}

ordinairement sur un conduit d'aqueduc. Surtout, elle résulte d'une volonté de l'hydraulicien qui l'a conçue comme le montre la prise d'eau: le relèvement du plancher de la dérivation à son départ ne peut pas s'expliquer par une erreur de nivellement. À partir de ces données et en appliquant la formule de Manning-Strickler, S. Heimann a évalué le débit maximal potentiel à $1350 \mathrm{l} / \mathrm{s}$. Ce chiffre approche celui qui avait été proposé par C.-L. Sagui (1948). Mais, compte tenu des rugosités de la paroi, des dépôts de calcaire et des problèmes d'écoulement, il ramène le débit moyen à $260 \mathrm{l} / \mathrm{s}$, soit $22464 \mathrm{~m}^{3}$ /jour. Nettement en dessous de l'évaluation de Sagui ( $1000 \mathrm{l} / \mathrm{s})$, ce chiffre est proche de ceux que proposaient déjà N. Schnitter : 240 l/s et R. H. J. Sellin : 300 l/s (Schnitter, 1972 ; Sellin, 1981).

$$
*
$$

Les techniques mises en œurre dans la construction de l'aqueduc de Barbegal l'individualisent nettement par rapport au pont de l'aqueduc d'Arles. Certes, elles ne présentent pas un grand intérêt au point de vue strictement architectural. Ces ouvrages ne se distinguent guère des kilomètres de maçoneries de ce type, mises en œuvre de manière répétitive, que les ingénieurs romains ont su édifier dans les campagnes. Cependant, les $200 \mathrm{~m}$ d'arches du pont-aqueduc de Barbegal constituent un jalon dans l'histoire des techniques de construction et contribuent à faire du vallon des Arcs un site où l'on peut observer de manière juxtaposée les divers appareils constructifs mis en œuvre durant plus de quatre siècles par les constructeurs romains. Cela en complète l'intérêt patrimonial.

Ces ouvrages présentent un autre intérêt, le principal à nos yeux. Ce pont est un ouvrage de jonction entre deux autres: l'aqueduc d'Arles et les moulins de Barbegal dont les fouilles ont montré qu'ils avaient l'un et l'autre une histoire plus longue et plus complexe que ce que l'on attendait. Sa conservation est médiocre. Cependant l'arche $B 4 / B 5$ témoigne d'une réfection tardive qui ne surprend pas, compte tenu des observations faites sur la section du canal comprise entre le bassin et la dérivation (voir J.-L. Guendon, supra, p. 96) et sur l'exutoire des moulins (Leveau et al., 2000). Après probablement trois quarts de siècle ou un siècle de fonctionnement optimal, les moulins, dont cet ouvrage assurait l'alimentation, ont connu un fonctionnement partiel ou occasionnel. Dans sa banalité, il apporte un témoignage exceptionnel sur une situation mal documentée, mais qui devait être relativement fréquente: la modification et l'évolution d'un système hydraulique en réponse à un changement d'utilisation, ici un besoin nouvellement apparu, l'alimentation des moulins construits à Barbegal dans un site propice à l'aménagement d'une chute d'eau. Ce facteur paraît avoir joué un rôle déterminant, et non l'existence d'un vaste plan d'eau dans la vallée des Baux à laquelle on pouvait croire avant les récentes recherches archéologiques et géomorphologiques (Leveau et al., 2002). 